\title{
Stability of the stationary solutions of the Allen-Cahn equation with non-constant stiffness
}

\author{
Paolo Buttà $^{\mathrm{a}}$, Emilio N.M. Cirillo ${ }^{\mathrm{b}}$, Giulio Sciarra ${ }^{\mathrm{c}}$ \\ ${ }^{a}$ Dipartimento di Matematica, Sapienza Università di Roma, piazzale Aldo Moro 5, \\ I-00185, Roma, Italy. \\ ${ }^{b}$ Dipartimento di Scienze di Base e Applicate per l'Ingegneria, Sapienza Università di \\ Roma, via A. Scarpa 16, I-00161, Roma, Italy. \\ ${ }^{c}$ Institut de Recherche en Génie Civil et Mécanique, Ecole Centrale de Nantes, 1, rue de la \\ Noë 44321 Nantes, France
}

\begin{abstract}
We study the solutions of a generalized Allen-Cahn equation deduced from a Landau energy functional, endowed with a non-constant higher order stiffness. We assume the stiffness to be a positive function of the field and we discuss the stability of the stationary solutions proving both linear and local non-linear stability.
\end{abstract}

Keywords: Allen-Cahn equation, gradient equation, phase coexistence, interface, stability

2010 MSC: 74A50; 35B35; 76S05

\section{Introduction}

The behavior of homogeneous phases simoultaneously present in a physical system can be described via a phase-field $\phi(x, t)$ depending on the space variable $x \in \Omega \subset \mathbb{R}^{3}$ and on the time variable $t \in[0, \infty)$. Two particular values 5 of the field represent the two homogeneous phases. These models have been widely used in the study of the spinodal decomposition phenomenon [1, 2, [3, namely, the process in which a system undergoing a second order phase transition is suddenly quenched from the disordered high temperature phase into a

Email addresses: butta@mat.uniroma1.it (Paolo Buttà), emilio.cirillo@uniroma1.it (Emilio N.M. Cirillo), giulio.sciarra@ec-nantes.fr (Giulio Sciarra)

Preprint submitted to Wave Motion

August 3, 2020 
broken-symmetry low temperature state and the evolving field $\phi(x, t)$ encodes the process of separation of the low temperature phases [4.

The evolution equation can be obtained as a gradient equation [5, 6] of a suitable Landau energy functional, namely,

$$
\frac{\partial \phi}{\partial t}=-\operatorname{grad} H(\phi) \quad \text { with } \quad H(\phi):=\int_{\Omega}\left[\frac{1}{2} \varepsilon|\nabla \phi|^{2}+W(\phi)\right] \mathrm{d} x,
$$

where the symbol grad means the variational derivative of the functional $H$. The function $W \in C^{2}(\mathbb{R})$ is the bulk energy of the field $\phi$ and the higher-order stiffness (or, simply, stiffness) $\varepsilon$ weights the interface contribution represented by the squared-gradient term. The bulk energy $W$ is usually chosen as a a double well with the two minima corresponding to the two phases 0 and 1 . If the stiffness is constant and no constraint to the total value of the field $\phi$ is imposed, the standard Allen-Cahn or Ginzburg-Landau equation

$$
\frac{\partial \phi}{\partial t}=\varepsilon \Delta \phi-W^{\prime}(\phi)
$$

is found, see, e.g., [7, which was firstly introduced to describe the motion of anti-phase boundaries in crystalline solids. Recently a similar model has been introduced also in the framework of the mechanics of (partially) saturated porous media, see, e.g., [8, 9, 10].

In this paper we consider the case in which the higher-order stiffness is not constant [11, 12, 13, but it is a sufficiently regular positive function of the field, namely, $\varepsilon \in C^{\infty}(\mathbb{R})$ such that there is $\bar{C}>1$ for which

$$
\overline{\bar{C}} \leq \varepsilon(\phi) \leq \bar{C} .
$$

The fact that $\varepsilon$ is strictly positive will be crucial in this paper, indeed, it has been proven in different contexts, see, e.g., [13, 14, 15, that if the stiffness is allowed to vanish in correspondence of the homogeneous states, not regular stationary solutions, often called compactons [16, can be found.

We also assume that the double well function $W \in C^{\infty}(\mathbb{R})$ and satisfies, for 
some $\bar{r} \in(0,1)$,

$$
\begin{aligned}
& W(0)=W(1)=0, \\
& W^{\prime}(r)>0 \text { for } r \in(0, \bar{r}), \\
& W^{\prime}(r)<0 \text { for } r \in(\bar{r}, 1), \\
& W^{\prime \prime}(0)>0, \quad W^{\prime \prime}(1)>0 .
\end{aligned}
$$

In the not constant stiffness case, the gradient equation (1) provides the generalized Allen-Cahn equation

$$
\frac{\partial \phi}{\partial t}=\frac{1}{2} \varepsilon^{\prime}(\phi)|\nabla \phi|^{2}+\varepsilon(\phi) \Delta \phi-W^{\prime}(\phi)
$$

see, for instance, [13, Appendix A].

It is useful to reformulate the problem in terms of the field

$$
u(x, t):=g(\phi(x, t)), \quad g(\phi):=\int_{1 / 2}^{\phi} \sqrt{\varepsilon(s)} \mathrm{d} s .
$$

Indeed, a straightforward computation shows that the Landau energy functional (1) and the evolution equation (5) expressed in terms of the new field $u$ read,

$$
F(u)=\int_{\Omega}\left[\frac{1}{2}|\nabla u|^{2}+\tilde{W}(u)\right] \mathrm{d} x
$$

and

$$
\frac{\partial u}{\partial t}=-\sigma(u) \operatorname{grad} F(u)=\sigma(u)\left[\Delta u-\tilde{W}^{\prime}(u)\right],
$$

${ }_{20}$ where $\tilde{W}:=W \circ g^{-1}$ is still double well function and $\sigma:=\varepsilon \circ g^{-1}$ plays the role of a mobility for the dynamics.

In the sequel, we shall consider the problem formulated in terms of the field $u$. Moreover, via an affine transformation of $u$, we can assume that the double well function, again denoted by $W$, still assumes its minima in $u=0,1$ and equations (4) hold (with a different $\bar{r}$ ).

We focus on the one-dimensional case $\Omega=\mathbb{R}$ and study the evolution problem

$$
\left\{\begin{array}{l}
\frac{\partial u}{\partial t}=\sigma(u)\left[\frac{\partial^{2} u}{\partial x^{2}}-W^{\prime}(u)\right] \\
u(x, t) \stackrel{x \rightarrow-\infty}{\longrightarrow} 0 \text { and } u(x, t) \stackrel{x \rightarrow+\infty}{\longrightarrow} 1
\end{array}\right.
$$




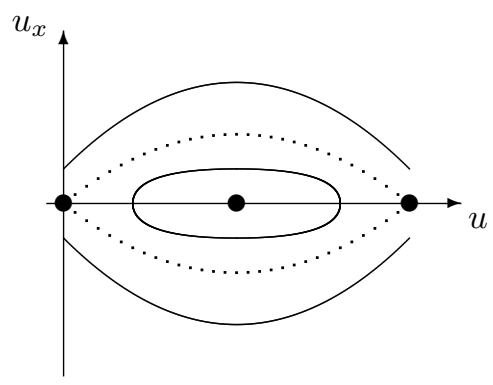

Figure 1: Phase portrait of the stationary equation 8. The dotted lines represent the separatrix.

namely, we solve the equation with Dirichlet boundary conditions 0 and 1 respectively at minus and plus infinity. As $\sigma>0$, the associated stationary problem reads,

$$
\left\{\begin{array}{l}
u^{\prime \prime}(x)-W^{\prime}(u(x))=0 \\
u(x) \stackrel{x \rightarrow-\infty}{\longrightarrow} 0 \text { and } u(x) \stackrel{x \rightarrow+\infty}{\longrightarrow} 1
\end{array}\right.
$$

and is often addressed to in the literature as the problem of finding a connection between the two phases on the line [17, 18.

The existence of the solution to the connection problem is an immediate consequence of a simple qualitative analysis of the equation (8) based on the conservation law,

$$
\frac{\mathrm{d}}{\mathrm{d} x}\left[\frac{1}{2}\left(u^{\prime}\right)^{2}-W(u)\right]=0 .
$$

It is easily seen that there exists a unique solution up to space translations, precisely the function $\bar{u}(x)$ defined by

$$
x=\int_{1 / 2}^{\bar{u}(x)} \sqrt{\frac{1}{2 W(s)}} \mathrm{d} s, \quad x \in \mathbb{R},
$$

together with its translates $\bar{u}_{z}(x)=\bar{u}(x-z)$. The phase portrait of the solutions to (9) in the interval $u \in[0,1]$ is plotted in figure 1 . The separatrix is represented so by a dotted line and its portion in the half-plane $u^{\prime}>0$ corresponds to the solution of the connection problem. 
In this paper we discuss the stability of such stationary solutions, hereafter named standing waves or fronts, following ideas developed in [19] in the case of the standard Allen-Cahan evolution, see, also, 20. We prove both linear stability in Section 2 and local non-linear stability in Section 3. Some numerical simulations will be finally discussed in the closing Section 4.

\section{Linear stability}

We first derive the linearized problem associated to (8). We consider the case of the reference solution $\bar{u}$, the adaptation to the case of its translates $\bar{u}^{z}$ 40 is immediate.

We first emphasize some relevant properties of the connection $\bar{u}$. In view of the assumptions on $W$ and $\sigma$, by $(10)$ it follows that $\bar{u} \in C^{\infty}(\mathbb{R})$ with $\bar{u}^{\prime}(x)>0$ for any $x \in \mathbb{R}$. Moreover, $\bar{u}$ approaches its limit exponentially fast as $x \rightarrow \pm \infty$; more precisely, there exist $A_{0}, A_{1}>0$ such that

$$
\lim _{x \rightarrow-\infty} \mathrm{e}^{-\alpha_{0} x} \bar{u}(x)=A_{0}, \lim _{x \rightarrow+\infty} \mathrm{e}^{\alpha_{1} x}(1-\bar{u}(x))=A_{1},
$$

where $\alpha_{0}=\sqrt{W^{\prime \prime}(0)}$ and $\alpha_{1}=\sqrt{W^{\prime \prime}(1)}$.

Finally, as $\bar{u}^{\prime}=\sqrt{2 W(\bar{u})}$, by (11) a similar estimate holds for the derivative:

$$
\lim _{x \rightarrow-\infty} \mathrm{e}^{-\alpha_{0} x} \bar{u}^{\prime}(x)=A_{0}^{\prime}, \quad \lim _{x \rightarrow+\infty} \mathrm{e}^{\alpha_{1} x} \bar{u}^{\prime}(x)=A_{1}^{\prime},
$$

with $A_{0}^{\prime}=A_{0} \sqrt{W^{\prime \prime}(0)}$ and $A_{1}^{\prime}=A_{1} \sqrt{W^{\prime \prime}(1)}$.

Letting $u=\bar{u}+v$ and using that $\bar{u}$ is a stationary solution, to the first order in $v$ the problem $(7)$ yields,

$$
\left\{\begin{array}{l}
\frac{\partial v}{\partial t}=\sigma(\bar{u})\left[\frac{\partial^{2} v}{\partial x^{2}}-W^{\prime \prime}(\bar{u}) v\right] \\
v(x, t) \stackrel{x \rightarrow-\infty}{\longrightarrow} 0 \text { and } v(x, t) \stackrel{x \rightarrow+\infty}{\longrightarrow} 0
\end{array}\right.
$$

By using again that $\bar{u}$ is a solution of the stationary problem, it is possible to cancel the term depending on the potential energy $W$ in the linearized problem. Indeed, by differentiating the stationary equation (8) we have

$$
\bar{u}^{\prime \prime \prime}(x)=W^{\prime \prime}(\bar{u}(x)) \bar{u}^{\prime}(x) .
$$


By substituting this expression in the equation 13 we have that the linearized equation can be written as

$$
\frac{\partial v}{\partial t}=L v
$$

with $L$ the linear operator

$$
L=a(x)\left[\frac{\mathrm{d}^{2}}{\mathrm{~d} x^{2}}-\frac{\bar{u}^{\prime \prime \prime}(x)}{\bar{u}^{\prime}(x)}\right], \quad a(x):=\sigma(\bar{u}(x)),
$$

on the space $L^{2}(\mathbb{R} ; \mathrm{d} x) \cap C^{2}(\mathbb{R})$ or $C_{b}(\mathbb{R}) \cap C^{2}(\mathbb{R})$, where $C_{b}(\mathbb{R})$ is the space of bounded continuous functions. We remark that, by the assumptions on $\sigma$, the

45 function $a(x)$ is smooth, bounded, and such that $\inf _{x} a(x)>0$.

By (14) it follows that $\bar{u}^{\prime}$ is an eigenfunction of $L$ with eigenvalue zero. Our task is to show that the rest of the spectrum is strictly negative. To this end, we take advantage of the fact that $\bar{u}^{\prime}>0$ to reduce the problem to estimating the rate of convergence to equilibrium of a suitable diffusion process.

We notice that $L \bar{u}^{\prime}=\bar{u}^{\prime} \mathcal{L}$, where

$$
\mathcal{L}=a(x)\left[\frac{\mathrm{d}^{2}}{\mathrm{~d} x^{2}}-U^{\prime}(x) \frac{\mathrm{d}}{\mathrm{d} x}\right]
$$

with

$$
U(x)=-2 \log \bar{u}^{\prime}(x) .
$$

The operator $\mathcal{L}$ is the generator of a diffusion. More precisely, it is the generator of the Markov semigroup associated to the dynamics on $\mathbb{R}$ given by the solution to the stochastic differential equation,

$$
\mathrm{d} x=a(x) U^{\prime}(x) \mathrm{d} t+\sqrt{2 a(x)} \mathrm{d} B
$$

where $B=B(t)$ is a 1-dimensional Wiener process. Since $a>0$, by elliptic regularity the transition probability $P_{t}(x, \mathrm{~d} y)$ admits a smooth density $p_{t}(x, y)$ which is the fundamental solution associated to $\mathcal{L}$, i.e.,

$$
\left\{\begin{array}{l}
\frac{\partial p_{t}}{\partial t}(x, \cdot)=\mathcal{L} p_{t}(x, \cdot) \\
p_{0}(x, y)=\delta_{y}(x)
\end{array}\right.
$$

50 Moreover, $p_{t}(x, y)>0$ for any $x, y \in \mathbb{R}$ and $t>0$, hence the associated Markov process is irreducible aperiodic. 
Under these conditions, the process has a unique (up to multiplication with a constant) invariant measure with a smooth positive density [21]. This density is solution to the stationary Fokker-Plank equation $\mathcal{L}^{*} \varrho=0$, where $\mathcal{L}^{*}$ is the $L^{2}$-adjoint of $\mathcal{L}$. An explicit computation shows that $\varrho(x)=\bar{u}^{\prime}(x)^{2} a(x)^{-1}=$ $\mathrm{e}^{-U(x)} a(x)^{-1}$. By 12 , $\varrho$ is integrable so that we obtain an invariant probability measure,

$$
\mu(\mathrm{d} x)=\frac{\bar{u}^{\prime}(x)^{2} a(x)^{-1} \mathrm{~d} x}{\int_{\mathbb{R}} \bar{u}^{\prime}(x)^{2} a(x)^{-1} \mathrm{~d} x^{\prime}} .
$$

It is worthwhile to notice that the process is in fact reversible with respect to $\mu$, since by integration by parts,

$$
\int \psi \mathcal{L} \varphi \mathrm{d} \mu=\int(\mathcal{L} \psi) \varphi \mathrm{d} \mu=-\int \sigma(\bar{u}) \psi^{\prime} \varphi^{\prime} \mathrm{d} \mu .
$$

To study the convergence $P_{t} \rightarrow \mu$ as $t \rightarrow \infty$ we apply a classical result based on the existence of a Liapunov function. By definition, a Liapunov function is a function $V \geq 1$ with compact level sets (i.e., $V(x) \rightarrow+\infty$ as $|x| \rightarrow \infty$ ). We claim there exists a Liapunov function $V$ for which there are a compact set $K \subset \mathbb{R}$ and constants $r>0$ and $b<\infty$ such that

$$
\mathcal{L} V(x) \leq-r V(x)+b \mathbb{1}_{K}(x) .
$$

Then, see, e.g., [21, Thm. 8.7], introducing the weighted norms,

$$
\|\varphi\|_{V, \infty}=\sup _{x \in \mathbb{R}} \frac{|\varphi(x)|}{V(x)}, \quad\|\varphi\|_{V, p}=\left\|\frac{\varphi}{V}\right\|_{L^{p}(\mathbb{R} ; \mathrm{d} x)}
$$

and letting

$$
P_{t} \varphi(x)=\int p_{t}(x, y) \varphi(y) \mathrm{d} y, \quad \mu(\varphi)=\int \varphi \mathrm{d} \mu,
$$

there are constants $C>0$ and $\alpha>0$ such that the bounds

$$
\begin{aligned}
\left\|P_{t} \varphi-\mu(\varphi)\right\|_{V} & \leq C \mathrm{e}^{-\alpha t}\|\varphi-\mu(\varphi)\|_{V}, \\
\left\|P_{t} \varphi-\mu(\varphi)\right\|_{V, p} & \leq C \mathrm{e}^{-\alpha t}\|\varphi-\mu(\varphi)\|_{V, p}
\end{aligned}
$$

hold for every measurable function $\varphi$ with finite weighted norm.

We now prove the claim on the existence of $V$ for which 20 holds. By 12 , the function $V=\gamma \mathrm{e}^{U / 2}=\gamma\left(\bar{u}^{\prime}\right)^{-1}$, with $\gamma=\max \bar{u}^{\prime}$, is Liapunov and, by an 
explicit computation,

$$
\mathcal{L} V=\frac{a}{2}\left(U^{\prime \prime}-\frac{1}{2}\left(U^{\prime}\right)^{2}\right) V .
$$

Now, by (8), (14), in view of (11), and (12),

$$
\begin{aligned}
& \lim _{x \rightarrow-\infty} \frac{\bar{u}^{\prime \prime}(x)}{\bar{u}^{\prime}(x)}=\sqrt{W^{\prime \prime}(0)}, \quad \lim _{x \rightarrow+\infty} \frac{\bar{u}^{\prime \prime}(x)}{\bar{u}^{\prime}(x)}=\sqrt{W^{\prime \prime}(1)}, \\
& \lim _{x \rightarrow-\infty} \frac{\bar{u}^{\prime \prime \prime}(x)}{\bar{u}^{\prime}(x)}=W^{\prime \prime}(0), \quad \lim _{x \rightarrow+\infty} \frac{\bar{u}^{\prime \prime \prime}(x)}{\bar{u}^{\prime}(x)}=W^{\prime \prime}(1) .
\end{aligned}
$$

By computing $U^{\prime}$ and $U^{\prime \prime}$ and using the above limits, a straightforward computation shows,

$$
\begin{aligned}
\lim _{x \rightarrow-\infty} \frac{a(x)}{2}\left(U^{\prime \prime}(x)-\frac{1}{2} U^{\prime}(x)^{2}\right) & =-\sigma(0) W^{\prime \prime}(0), \\
\lim _{x \rightarrow+\infty} \frac{a(x)}{2}\left(U^{\prime \prime}(x)-\frac{1}{2} U^{\prime}(x)^{2}\right) & =-\sigma(1) W^{\prime \prime}(1) .
\end{aligned}
$$

Therefore, in view of (22), the function $V$ clearly satisfies $(20)$, e.g., with $r=$ $\frac{1}{2} W^{\prime \prime}(0) \wedge W^{\prime \prime}(1)$ and $K=[-R, R]$ so large that $V>4$ and $\frac{1}{2} a\left(U^{\prime \prime}-\frac{1}{2}\left(U^{\prime}\right)^{2}\right)>r$ outside $K$.

The desired spectral properties of the operator $L$ can be now easily deduced from (21). To this end, it is useful to work in the Hilbert space $L^{2}\left(\mathbb{R} ; a(x)^{-1} \mathrm{~d} x\right)$, where $L$ is symmetric. We denote the inner product by $\langle\cdot, \cdot\rangle_{a}$ and the corresponding norm by $\|\cdot\|_{a, L^{2}}$. We observe that since $V=\gamma\left(\bar{u}^{\prime}\right)^{-1}$, for every $v \in C_{b}(\mathbb{R})$ or $v \in L^{2}(\mathbb{R} ; \mathrm{d} x)$ the function $\varphi_{v}:=v / \bar{u}^{\prime}$ has the corresponding weighted norms finite. Moreover, as $\mathrm{e}^{L t}=\bar{u}^{\prime} P_{t}\left(\bar{u}^{\prime}\right)^{-1}$,

$$
\mathrm{e}^{L t} v-\langle v, q\rangle_{a} q=\left[P_{t} \varphi_{v}-\mu\left(\varphi_{v}\right)\right] \bar{u}^{\prime}
$$

where $q(x)=\left\|\bar{u}^{\prime}\right\|_{a, L^{2}}^{-1} \bar{u}^{\prime}(x)$ is the normalized eigenfunction of $L$ with eigenvalue zero. Therefore, by (21), it is easily deduced that there is a constant $C_{0}>0$ such that

$$
\begin{aligned}
\left\|\mathrm{e}^{L t} v-\langle v, q\rangle_{a} q\right\|_{L^{2}} & \leq C_{0} \mathrm{e}^{-\alpha t}\left\|v-\langle v, q\rangle_{a} q\right\|_{L^{2}}, \\
\left\|\mathrm{e}^{L t} v-\langle v, q\rangle_{a} q\right\|_{\infty} & \leq C_{0} \mathrm{e}^{-\alpha t}\left\|v-\langle v, q\rangle_{a} q\right\|_{\infty},
\end{aligned}
$$

where, in the first estimate, we used that, in view of the assumptions on $\sigma$, the norms $\|\cdot\|_{a, L^{2}}$ and $\|\cdot\|_{L^{2}}$ are equivalent. 
An estimate like 23 holds also with respect to the $H^{1}$-norm. This is quite standard but we sketch the proof for the sake of completeness. To this purpose, we shall use the following estimates on the fundamental solution $\mathrm{e}^{L t}(x, y)$, which can be deduced by exploiting the parametric method to construct the fundamental solution, see [22]. Letting

$$
\mathrm{e}^{L t}(x, y)=\sqrt{\frac{a(y)}{4 \pi t}} \exp \left(-\frac{a(y)(x-y)^{2}}{4 t}\right)+Q_{t}(x, y),
$$

for any $T>0$ and $\kappa>\sup _{x} a(x)$ there exists a constant $C_{T}>0$ such that, for any $t \in(0, T]$ and $x, y \in \mathbb{R}$,

$$
\begin{aligned}
Q_{t}(x, y) & \leq C_{T} \exp \left(-\kappa \frac{(x-y)^{2}}{4 t}\right), \\
\left|\frac{\partial Q_{t}(x, y)}{\partial x}\right| & \leq \frac{C_{T}}{\sqrt{t}} \exp \left(-\kappa \frac{(x-y)^{2}}{4 t}\right) .
\end{aligned}
$$

Let now $v \in H^{1}$ that, without loss of generality, we identify with its continuous representative. Clearly $\mathrm{e}^{L t} v-\langle v, q\rangle_{a} q=\mathrm{e}^{L t} v_{\perp}$, where $v_{\perp}=v-\langle v, q\rangle_{a} q$ is ${ }_{60}$ the component of $v$ orthogonal to $q$ in $L^{2}\left(\mathbb{R} ; a(x)^{-1} \mathrm{~d} x\right)$. We estimate $\left\|\mathrm{e}^{L t} v_{\perp}\right\|_{H^{1}}$ by analyzing separately the case $t \in(0,1]$ and $t>1$. In view of 23$)$, we only need to study the $L^{2}$-norm of $\frac{\partial\left(\mathrm{e}^{L t} v_{\perp}\right)}{\partial x}$.

If $t \in(0,1]$, in view of the identity

$$
\frac{\partial}{\partial x} \frac{\mathrm{e}^{-\frac{a(y)(x-y)^{2}}{4 t}}}{\sqrt{4 \pi a(y) t}}+\frac{\partial}{\partial y} \frac{\mathrm{e}^{-\frac{a(y)(x-y)^{2}}{4 t}}}{\sqrt{4 \pi a(y) t}}=\frac{a^{\prime}(y) \mathrm{e}^{-\frac{a(y)(x-y)^{2}}{4 t}}}{a(y) \sqrt{4 \pi a(y) t}}\left(\frac{1}{2}-\frac{a(y)^{2}(x-y)^{2}}{4 t}\right),
$$

by 24 and an integration by parts we get,

$$
\begin{aligned}
& \frac{\partial\left(\mathrm{e}^{L t} v_{\perp}\right)(x)}{\partial x}=\int \frac{\mathrm{e}^{-\frac{a(y)(x-y)^{2}}{4 t}}}{\sqrt{4 \pi a(y) t}} v_{\perp}^{\prime}(y) \mathrm{d} y \\
& +\int \frac{a^{\prime}(y) \mathrm{e}^{-\frac{a(y)(x-y)^{2}}{4 t}}}{a(y) \sqrt{4 \pi a(y) t}}\left(\frac{a(y)^{2}(x-y)^{2}}{4 t}-\frac{1}{2}\right) v_{\perp}(y) \mathrm{d} y \\
& +\int \frac{\partial Q_{t}(x, y)}{\partial x} v_{\perp}(y) \mathrm{d} y .
\end{aligned}
$$

By $(25)_{1}$, for some positive constants $C_{1}$ and $\lambda$,

$$
\left|\frac{\partial\left(\mathrm{e}^{L t} v_{\perp}\right)(x)}{\partial x}\right| \leq \int \frac{C_{1} \mathrm{e}^{-\frac{(x-y)^{2}}{4 \lambda t}}}{\sqrt{4 \pi \lambda t}}\left(\left|v_{\perp}(y)\right|+\left|v_{\perp}^{\prime}(y)\right|\right) \mathrm{d} y
$$


which implies, recalling that the heat kernel is a contraction in $L^{2}$,

$$
\left\|\frac{\partial\left(\mathrm{e}^{L t} v_{\perp}\right)}{\partial x}\right\|_{L^{2}} \leq C_{1}\left\|\left(\left|v_{\perp}\right|+\left|v_{\perp}^{\prime}\right|\right)\right\|_{L^{2}} \leq 2 C_{1}\|v\|_{H^{1}} .
$$

If $t>1$ we write,

$$
\frac{\partial\left(\mathrm{e}^{L t} v_{\perp}\right)(x)}{\partial x}=\int \frac{\partial \mathrm{e}^{L}(x, y)}{\partial x}\left(\mathrm{e}^{L(t-1)} v_{\perp}\right)(y) \mathrm{d} y
$$

and observe that, for any $f \in L^{2}(\mathbb{R} ; \mathrm{d} x)$, by 24 and 25$)_{1}$ for $t=1$, for some $C_{2}>0$,

$$
\left\|\frac{\partial\left(\mathrm{e}^{L} f\right)}{\partial x}\right\|_{L^{2}} \leq C_{2}\|f\|_{L^{2}} .
$$

Therefore, in view of 23$)_{1}$, setting $C_{3}=C_{0} C_{2}$,

$$
\left\|\frac{\partial\left(\mathrm{e}^{L t} v_{\perp}\right)}{\partial x}\right\|_{L^{2}} \leq C_{2}\left\|\mathrm{e}^{L(t-1)} v_{\perp}\right\|_{L^{2}} \leq C_{3} \mathrm{e}^{-\alpha(t-1)}\left\|v_{\perp}\right\|_{L^{2}} \leq C_{3} \mathrm{e}^{-\alpha t}\left\|v_{\perp}\right\|_{H^{1}} .
$$

In conclusion, there is a constant $C_{4}>0$ such that

$$
\left\|\mathrm{e}^{L t} v-\langle v, q\rangle_{a} q\right\|_{H^{1}} \leq C_{4} \mathrm{e}^{-\alpha t}\left\|v-\langle v, q\rangle_{a} q\right\|_{H^{1}} .
$$

\section{Non-linear stability}

In this section we prove the one-dimensional manifold $\mathcal{M}:=\left\{\bar{u}_{z}: z \in \mathbb{R}\right\}$ of the translates of $\bar{u}$ is asymptotically stable. This is the content of two theorems.

The first result follows from the linear analysis done in the previous section. Indeed, $\lambda=0$ is a semisimple eigenvalue of the (unbounded) operator $L$ on $H^{1}$, and the estimate 26) shows further that the remaining part of the spectrum is strictly contained in $\{\lambda: \Re \lambda<0\}$. This implies, see, e.g., [23, Proposition 4.1], 70 the following stability theorem.

Theorem 1. Consider the Cauchy problem

$$
\left\{\begin{array}{l}
\frac{\partial u}{\partial t}=\sigma(u)\left[\frac{\partial^{2} u}{\partial x^{2}}-W^{\prime}(u)\right], \\
u(x, 0)=u_{0}(x) .
\end{array}\right.
$$

For any $\varepsilon>0$ there exists $\delta>0$ such that if the initial datum $u_{0}$ satisfies $\left\|u_{0}-\bar{u}_{\zeta_{0}}\right\|_{H^{1}} \leq \delta$ for some $\zeta_{0} \in \mathbb{R}$ then the solution $u(t)=u(\cdot, t)$ exists for all 
$t \geq 0$ and $\operatorname{dist}_{H^{1}}(u(t), \mathcal{M}) \leq \varepsilon$ for any $t \geq 0$. Moreover, if $\delta$ is small enough, there is $z_{0} \in \mathbb{R}$ such that, for some $\beta>0$,

$$
\lim _{t \rightarrow+\infty} \mathrm{e}^{\beta t}\left\|u(t)-\bar{u}_{z_{0}}\right\|_{H^{1}}=0
$$

and $\left|z_{0}-\zeta_{0}\right| \rightarrow 0$ if $\delta \rightarrow 0$.

In Theorem 2 below, we instead prove convergence in sup-norm to a suitable front of those solutions whose initial datum approximately resembles a front. The proof is an adaptation of the argument in the seminal paper [19, where the case of constant mobility is concerned. In [19] it is also shown that the limit is reached exponentially fast in time. Due to the non-constant mobility, we are able to prove this rate of convergence only under the more restrictive assumption on the initial datum of Theorem 1

Theorem 2. Consider the Cauchy problem Eq. 27) and assume that $u_{0}$ is a piecewise continuous function such that $0 \leq u_{0} \leq 1$. Then, there exists a unique bounded classical solution $u(x, t)$ and $0 \leq u(x, t) \leq 1$. Furthermore, if

$$
\limsup _{x \rightarrow-\infty} u_{0}(x)<\bar{r}, \liminf _{x \rightarrow+\infty} u_{0}(x)>\bar{r},
$$

with $\bar{r}$ as in (4), there is $z_{0} \in \mathbb{R}$ such that

$$
\lim _{t \rightarrow+\infty}\left\|u(\cdot, t)-\bar{u}_{z_{0}}\right\|_{\infty}=0 .
$$

Proof. We split the proof into three steps.

so Step 1: Existence and uniqueness. The existence and uniqueness in large of bounded classical solution $u(x, t)$ and that $0 \leq u(x, t) \leq 1$ follow from a priori estimates and comparison theorems for quasilinear parabolic equations, see, e.g., 24.

Step 2: A priori estimates. There are $z_{1}, z_{2} \in \mathbb{R}$ and $q_{0}, \mu>0$ such that

$$
\bar{u}_{z_{1}}(x)-q_{0} \mathrm{e}^{-\mu t} \leq u(x, t) \leq \bar{u}_{z_{2}}(x)+q_{0} \mathrm{e}^{-\mu t} .
$$

We prove the lower bound, the other can be obtained similarly. We fix $q_{0}>0$ such that

$$
q_{0}<\bar{r}<1-q_{0}<\liminf _{x \rightarrow+\infty} u_{0}(x)
$$


so that there exists $z_{*}$ large enough for which $\bar{u}_{z_{*}}(x)-q_{0} \leq u_{0}(x)$ for any $x \in \mathbb{R}$. We then look for $z_{1}>z_{*}$ and $\mu>0$ such that, setting

$$
q(t)=q_{0} \mathrm{e}^{-\mu t}, \quad z(t)=z_{1}+\left(z_{*}-z_{1}\right) \mathrm{e}^{-\mu t},
$$

the function

$$
u_{-}(x, t):=\max \left\{0 ; \bar{u}_{z(t)}(x)-q(t)\right\}
$$

is a subsolution. This clearly proves the estimate since $u_{-}(x, t) \leq u(x, t)$ by the ${ }_{85}$ Comparison Theorem and $\bar{u}_{z_{1}}(x)-q_{0} \mathrm{e}^{-\mu t} \leq u_{-}(x, t)$ by construction.

Since $u=0$ is a solution, $u_{-}$is a subsolution if $u_{-}>0$ implies

$$
N\left[u_{-}\right]:=\frac{\partial u_{-}}{\partial t}-\sigma\left(u_{-}\right)\left[\frac{\partial^{2} u_{-}}{\partial x^{2}}-W^{\prime}\left(u_{-}\right)\right] \leq 0 .
$$

Now, if $u_{-}>0$, we have $\bar{u}_{z}>q$ and

$$
N\left[u_{-}\right]=-\dot{z} \bar{u}_{z}^{\prime}-\dot{q}+\sigma\left(\bar{u}_{z}-q\right)\left[W^{\prime}\left(\bar{u}_{z}-q\right)-W^{\prime}\left(\bar{u}_{z}\right)\right],
$$

where we used that $\bar{u}_{z}^{\prime \prime}=W^{\prime}\left(\bar{u}_{z}\right)$ and omitted the explicit dependence on $x$ and $t$. Since $q_{0}<\bar{r}<1-q_{0}, W^{\prime}(0)=W^{\prime}(1)=0$, and $W^{\prime \prime}(0)$ and $W^{\prime \prime}(1)$ are positive, there is $C>0$ such that $W^{\prime}(1-q)-W^{\prime}(1)<-2 C q$ and $W^{\prime}(0)-W^{\prime}(q) \leq-2 C q$ for any $q \in\left[0, q_{0}\right]$. Therefore, by continuity, there exists $\delta<q_{0}$ such that $W^{\prime}(u-q)-W^{\prime}(u) \leq-C q$ for any $(u, q) \in[1-\delta, 1] \times\left[0, q_{0}\right]$ and for any $0 \leq q \leq u \leq \delta$. We conclude that whenever $u_{z} \in[1-\delta, 1]$ or $q \leq \bar{u}_{z} \leq \delta$ we have,

$$
N\left[u_{-}\right] \leq-\dot{z} \bar{u}_{z}^{\prime}-\dot{q}-(\bar{C} / C) q \leq(\mu-\bar{C} / C) q,
$$

with $\bar{C}$ as in Eq. (3) and where we used that $\dot{q}=-\mu q, \dot{z}>0$, and $\bar{u}_{z}^{\prime}>0$. On the other hand, from the properties of $\bar{u}$ and the smoothness of $W$, there is $C_{5}>0$ such that $\bar{u}^{\prime} \geq C_{5}$ whenever $\delta \leq \bar{u} \leq 1-\delta$ and $W^{\prime}\left(\bar{u}_{z}-q\right)-W^{\prime}\left(\bar{u}_{z}\right) \leq C_{5} q$ for any $q \in\left[0, q_{0}\right]$. Therefore, if $\delta \leq \bar{u}_{z} \leq 1-\delta$,

$$
N\left[u_{-}\right] \leq-C_{5} \dot{z}-\dot{q}+\bar{C} C_{5} q \leq\left[C_{5} \mu\left(z_{*}-z_{1}\right)+\left(\mu+\bar{C} C_{5}\right) q_{0}\right] \mathrm{e}^{-\mu t},
$$

where we used the upper bound in Eq. (3) and that $\dot{z}=-\mu\left(z_{*}-z_{1}\right) \mathrm{e}^{-\mu t}$. Therefore, choosing $\mu=\bar{C} / C$ and $z_{1}=z_{*}+q_{0}\left(\mu+C_{5}\right) /\left(\mu C_{5}\right)$ we obtain $N\left[u_{-}\right] \leq$ 0 whenever $u_{-}>0$. 
An immediate corollary of the proof of Eq. 29 is the stability of fronts.

90 some $z_{0} \in \mathbb{R}$ and small $\varepsilon>0$. Then, it is possible to choose $q_{0}=O(\varepsilon)$ and $\left|z_{*}-z_{0}\right|=O(\varepsilon)$, so that also $\left|z_{1}-z_{0}\right|+\left|z_{2}-z_{0}\right|=O(\varepsilon)$. In conclusion, $\left|u(x, t)-\bar{u}_{z_{0}}(x)\right|=O(\varepsilon)$ independently of $x \in \mathbb{R}$ and $t \geq 0$.

Step 3: Convergence to fronts. We now prove that there exists $z_{0} \in \mathbb{R}$ such that, uniformly in $x \in \mathbb{R}$,

$$
\lim _{t \rightarrow+\infty}\left|u(x, t)-\bar{u}_{z_{0}}(x)\right|=0 .
$$

We first observe that, for a suitable constant $C_{6}>0$,

$$
|1-u(x, t)|+\left|\frac{\partial u}{\partial t}(x, t)\right|+\left|\frac{\partial u}{\partial x}(x, t)\right|+\left|\frac{\partial^{2} u}{\partial x^{2}}(x, t)\right| \leq C_{6}\left(\mathrm{e}^{-\alpha_{1} x}+\mathrm{e}^{-\mu t}\right),
$$

for $x>0, t \geq 0$, and

$$
|u(x, t)|+\left|\frac{\partial u}{\partial t}(x, t)\right|+\left|\frac{\partial u}{\partial x}(x, t)\right|+\left|\frac{\partial^{2} u}{\partial x^{2}}(x, t)\right| \leq C_{6}\left(\mathrm{e}^{\alpha_{0} x}+\mathrm{e}^{-\mu t}\right),
$$

for $x<0$ and $t \geq 0$. The estimates in Eqs. (31) and (32) concerning the undifferentiated function $u$ come from Eqs. (11) and 29). Since $W^{\prime}(0)=W^{\prime}(1)=0$, these estimates also imply that $\left|W^{\prime}(u(x, t))\right| \leq C\left(\mathrm{e}^{\alpha_{0} x}+\mathrm{e}^{-\mu t}\right)$ for some $C>0$. The bounds on the differentiated function then follow from standard Schauder estimates for quasilinear parabolic equations, see, e.g., 24].

Then, by arguing exactly as in [19, Lemma 4.4], we conclude that for each $\delta>0$ the orbit $\{u(\cdot, t): t \geq \delta\}$ is relatively compact in $C^{2}(\mathbb{R})$.

To prove Eq. (30), we let $v$ be the following truncation of $u$,

$$
v(x, t)= \begin{cases}u(x, t) & \text { for }|x| \leq t \\ 0 & \text { for } x \leq-t-1 \\ 1 & \text { for } x \geq t+1\end{cases}
$$

where the interpolation is done smoothly in such a way that the estimates Eqs. (31) and (32) hold also for $v$ (possibly, with a larger constant $C_{6}$ ).

We next introduce the Lyapunov function

$$
F(v)=\int_{\mathbb{R}}\left[\frac{1}{2}\left|\frac{\partial v}{\partial x}\right|^{2}+W(v)\right] \mathrm{d} x .
$$


It is bounded uniformly in $t$ in view of Eqs. (31), (32), and since $W(0)=$ $W(1)=0$. Moreover, letting $f(t):=F(v(\cdot, t))$, after integration by parts it is easily shown that $f$ is differentiable and

$$
\dot{f}(t)=-\int_{\mathbb{R}}\left(\frac{\partial^{2} v}{\partial x^{2}}-W^{\prime}(v)\right) \frac{\partial v}{\partial t} \mathrm{~d} x .
$$

From the definition of $v$ it follows that $\dot{f}(t)=Q(t)+R(t)$ with

$$
Q(t)=-\int_{\mathbb{R}} \sigma(v)\left(\frac{\partial^{2} v}{\partial x^{2}}-W^{\prime}(v)\right)^{2} \mathrm{~d} x \leq 0
$$

and, for a some $C_{7}>0$,

$$
\begin{aligned}
|R(t)| & =\left|\int_{t \leq|x| \leq t+1}\left(\frac{\partial^{2} v}{\partial x^{2}}-W^{\prime}(v)\right) \frac{\partial v}{\partial t} \mathrm{~d} x\right| \\
& \leq C_{7} \int_{-t-1}^{-t}\left(\mathrm{e}^{\alpha_{0} x}+\mathrm{e}^{-\mu t}\right)^{2} \mathrm{~d} x+C_{7} \int_{t}^{t+1}\left(\mathrm{e}^{-\alpha_{1} x}+\mathrm{e}^{-\mu t}\right)^{2} \mathrm{~d} x .
\end{aligned}
$$

Therefore $\lim _{t \rightarrow+\infty} R(t)=0$ so that $\limsup _{t \rightarrow+\infty} \dot{f}(t) \leq 0$. Hence, since $f(t)$ is bounded uniformly in time, there must exist a diverging sequence $\left\{t_{n}\right\}$ such that $\lim _{n \rightarrow \infty} \dot{f}\left(t_{n}\right)=$ 0 . Since $\lim _{t \rightarrow+\infty} R(t)=0$, we finally obtain $\lim _{n \rightarrow \infty} Q\left(t_{n}\right)=0$.

Now, by compactness, we can extract a subsequence $\left\{t_{n_{k}}\right\}$ such that $u\left(\cdot, t_{n_{k}}\right)$, and hence also $v\left(\cdot, t_{n_{k}}\right)$, converges in $C^{2}(\mathbb{R})$ to some function $\bar{v}$. Since $\lim _{k \rightarrow \infty} Q\left(t_{n}\right)=$ 0 we deduce that

$$
\begin{aligned}
& \int_{|x| \leq K} \sigma(v)\left(\bar{v}^{\prime \prime}-W^{\prime}(\bar{v})\right)^{2} \mathrm{~d} x \\
& \quad=\lim _{k \rightarrow \infty} \int_{|x| \leq K} \sigma(v)\left(\frac{\partial^{2} v\left(\cdot, t_{n_{k}}\right)}{\partial x^{2}}-W^{\prime}\left(v\left(\cdot, t_{n_{k}}\right)\right)\right)^{2} \mathrm{~d} x \leq \lim _{k \rightarrow \infty} Q\left(t_{n}\right)=0
\end{aligned}
$$

for all $K>0$, so that $\bar{v}^{\prime \prime}-W^{\prime}(\bar{v})=0$. Since we also have $\bar{v}(-\infty)=0$ and $\bar{v}(+\infty)=1$, by the uniqueness of fronts we deduce $\bar{v}=\bar{u}_{z_{0}}$ for some $z_{0} \in \mathbb{R}$. To conclude the proof of Eq. (30), we simply recall that we have already proved the stability of fronts, so that the convergence of $u\left(\cdot, t_{n_{k}}\right)$ to $\bar{u}_{z_{0}}$ implies that of the whole trajectory $u(\cdot, t)$.

\section{Numerical check of stability results}

In this section we discuss the result of some numerical simulations aiming to check the stability properties stated in Theorems 1 and 2 . The strategy 
will be the following: i) we consider the particular solution $\bar{u} \in \mathcal{M}$ defined in Eq. (10) of the stationary equation Eq. (8) associated with the problem Eq. (7); ii) we consider a profile obtained by modifying locally $\bar{u}$ (type I) and a profile obtained by adding an oscillatory term to $\bar{u}$ in such a way that the assumptions of Theorem 2 are satisfied (type II); iii) the problem Eq. (7) is solved numerically via the finite elements method and the $H^{1}, L^{2}$, and $L^{\infty}$ norms of the difference between the solution at time $t$ and $t-\Delta t$ are computed as a function of time.

Our results will be plotted in figures made of two panels: in the left one we will show the solution of the problem Eq. (7) at different values of time; in the right panel the norm $\left(H^{1}, L^{2}\right.$, and $\left.L^{\infty}\right)$ of the difference between the solution at time $t$ and $t-\Delta t$. Different cases will correspond to different choices of the initial profile.

In this section the function $W$ is chosen as

$$
W(u)=\frac{1}{2} u^{2}\left(1-u^{2}\right),
$$

so that the stationary solution Eq. 10 is

$$
\bar{u}(x)=\frac{e^{x}}{1+e^{x}} .
$$

The stiffness function will be

$$
\sigma(u)=A+B \sin (2 \pi \nu u)
$$

with $A, B, \nu \geq 0$; note that for $\nu>1 / 2$ we shall choose $B<A$ so that the condition $\sigma(u)>0$ is safely satisfied. In the following picture we shall report results for the case $\nu=5 / 2$; other possible values of $\nu$ has been considered and similar results have been found.

The type I initial condition will be a continuous function obtained by substituting the $\bar{u}$ profile in the interval $[a, b]$ with an arc of parabola with vertex in $c$, with $a<c<b$. The type II initial condition will be a continuous function obtained by adding to $\bar{u}$ the function $D \sin [2 \pi \mu(x-d)]$ in the interval $[d, \infty)$, with $0<D \leq 1 / 4, d \geq 1$, and $\mu>0$. 

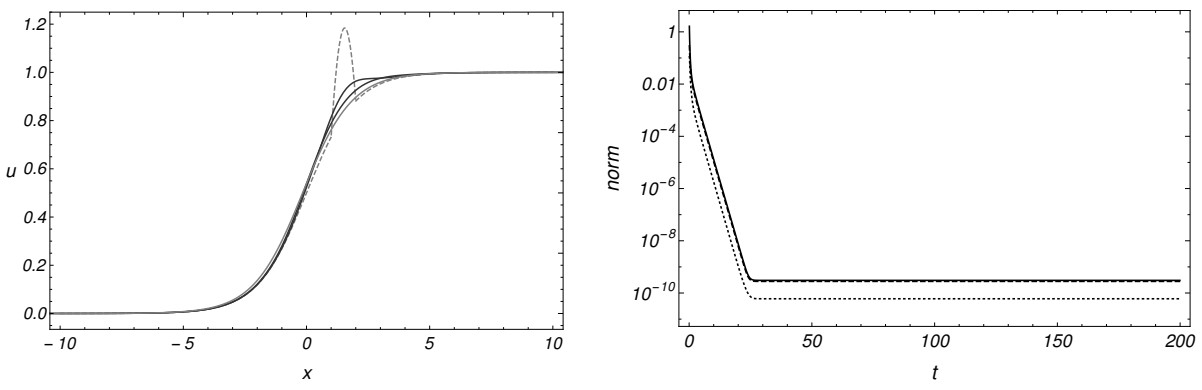

Figure 2: Solution of problem Eq. 77 for the initial condition of type I with $a=1, b=2$, and $c=1.55$ and stiffness Eq. 35 with $A=1, B=1 / 2$, and $\nu=5 / 2$. Left panel: profile $u(x, t)$ at times $t=0$ (dashed gray), 5 (solid black), 10 (solid black), and the stationary infinite time limit (solid gray). Right panel: semi-logarithmic plot of $H^{1}$ norm (solid line), $L^{2}$ norm (dashed line), $L^{\infty}$ norm (dotted line) of the difference between the solution at time $t$ and $t-\Delta t$.

Eq. (7) is solved via the finite element method powered with the NewtonRaphson algorithm over a finite domain. The boundary conditions are of Neumann type. The spatial discretization of the domain is based on quadratic shape functions with one hundred elements. As time discretization we use the backward Euler scheme with time step $\Delta t=1$. The algorithm is stopped when the $L^{2}$ norm of the difference between the current profile and the one computed one time step before is smaller than $10^{-4}$.

In Figure 2 an initial condition of type I is used (see the caption for more de-
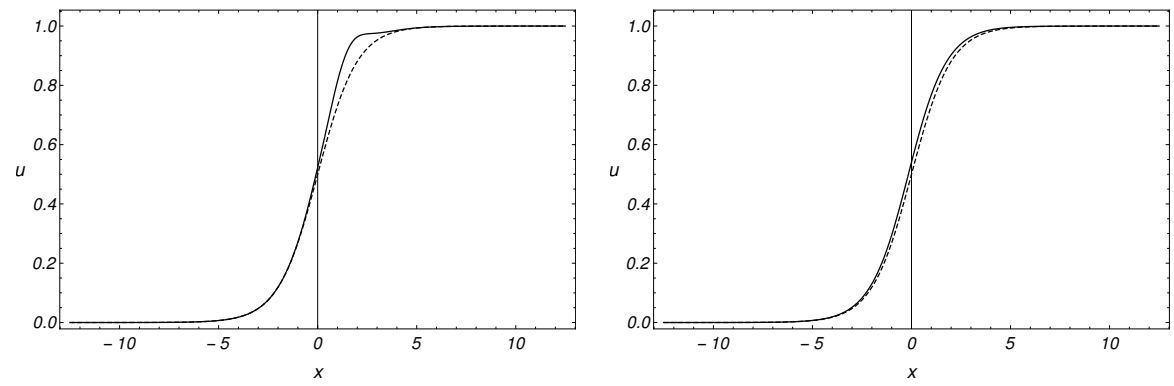

Figure 3: Solid lines represent the profiles at times $t=5$ (left) and $t=25$ (right) of the evolution described in Figure 2 The dashed line is the stationary profile already reported in Figure 2 

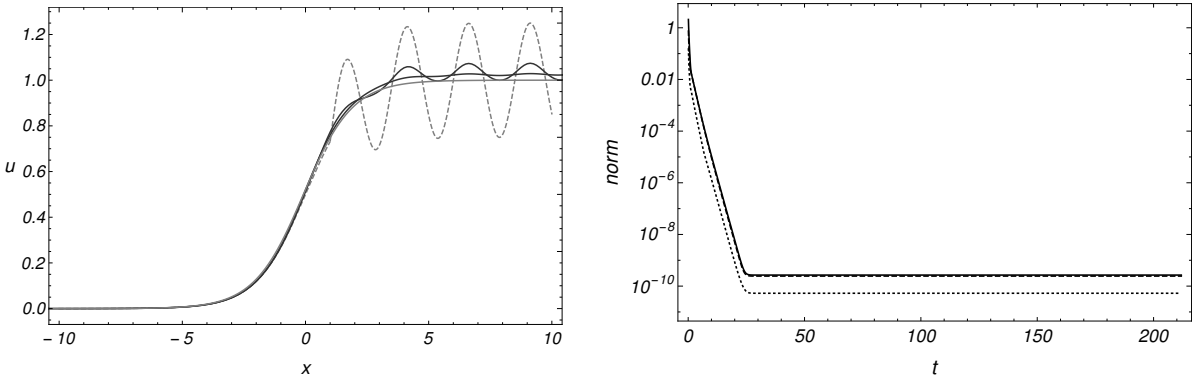

Figure 4: Solution of problem 77 for the initial condition of type II with $d=1, D=1 / 4$, and $\mu=0.4$ and stiffness Eq. 35 with $A=1, B=1 / 2$, and $\nu=5 / 2$. Left panel: profile $u(x, t)$ at times $t=0$ (dashed gray), 5 (solid black), 10 (solid black), and the stationary infinite time limit (solid gray). Right panel: semi-logarithmic plot of $H^{1}$ norm (solid line), $L^{2}$ norm (dashed line), $L^{\infty}$ norm (dotted line) of the difference between the solution at time $t$ and $t-\Delta t$.

tails) and, as already remarked, for the stiffness Eq. (35) we chose the frequency $\nu=5 / 2$. The solution of the equation converges to a solution in $\mathcal{M}$ slightly different from $\bar{u}$ which, we recall, has been perturbed to construct the initial condition. The behavior of the norm as a function of time is compatible with an exponential decay and show three different regimes. An initial quick drop is followed by a linear decrease and, finally, a long constant behavior onsets. As shown in Figure 3, where we report the profile corresponding to the times where the steepness of the norm changes abruptly, during the first part of the evolution the parabolic bump is removed, whereas in the second part the profile is slowly deformed to reach the stationary shape.

In Figure 4 an initial condition of type II is used (see the caption for more details) and for the stiffness Eq. 35 we chose, again, the frequency $\nu=5 / 2$. The solution of the equation quickly converges to a monotone interface profile with constant plateau lower than one. Then, the solution starts to increase the value of the plateau slowly approaching the correct limiting value one. The behavior of the norm as a function of time is compatible with an exponential decay, also in this case. The three regimes already noted in Figure 2 can be observed and explained as we did above. Indeed, as illustrated in Figure 5 in 

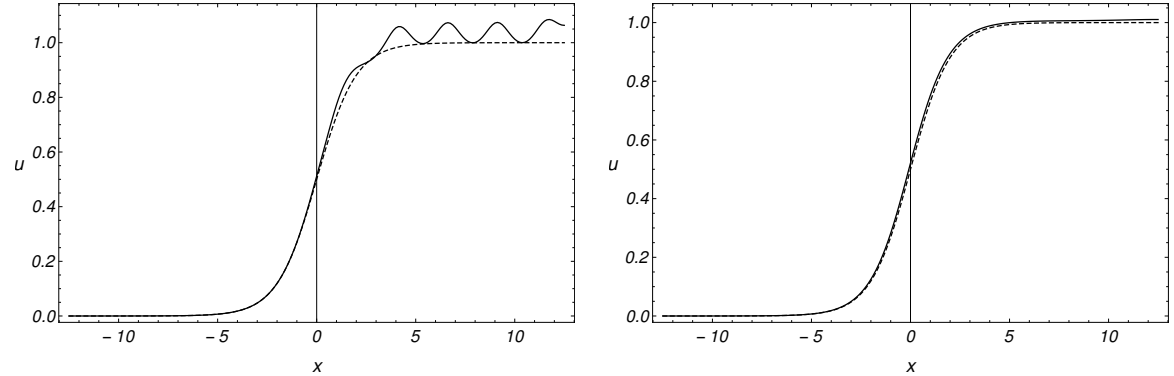

Figure 5: Solid lines represent the profiles at times $t=5$ (left) and $t=25$ (right) of the evolution described in Figure 4 . The dashed line is the stationary profile already reported in Figure 4
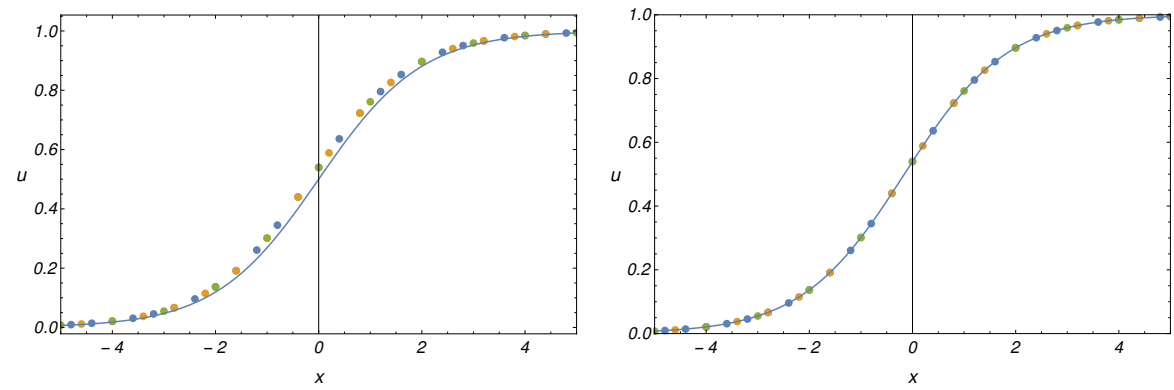

Figure 6: On the left, exact solution 34 and stationary profiles obtained for the simulation discussed in Figure 2 on the intervals $[-10,10]$ (yellow dots), $[-12.5,12.5]$ (blue dots), and $[-15,15]$ (green dots). On the right: the same, but the exact solution has been shifted by the amount 0.15881 for a perfect match.

the first part of the evolution, corresponding to the sudden drop of the norms, the ripples are removed and then the profile linearly converges to the stationary profile.

Finally, we note that our simulations have been performed on a finite interval, whereas the theoretical discussion of the previous sections refer to the evolution on the real infinite line. In Figure 6 we, thus, compare results obtained in simulations referring to different sizes of the space interval. Since, the stationary profiles obtained for the three different cases coincide (see the left panel), we can infer that our simulations capture, indeed, the infinite volume behavior.

Moreover, we also note that by suitably shifting the exact profile, as shown in 
the right panel, we obtain a perfect match with the stationary profiles obtained on the finite space interval.

\section{Acknowledgments}

ENMC thanks D. Andreucci for some very useful discussions and for having provided some references.

\section{References}

[1] A. J. Bray, Theory of phase-ordering kinetics, Advances in Physics 43 (1994) 357-459.

[2] J. S. Langer, Solids far from equilibrium, edited by C. Godrèche, Cambridge University Press, 1992, p. 297.

[3] D. J. Eyre, Systems of cahn-hilliard equations, SIAM Journal on Applied Mathematics 53 (1993) 1686-1712.

[4] E. N. M. Cirillo, M. Colangeli, E. Moons, A. Muntean, S.-A. Muntean, J. van Stam, A lattice model approach to the morphology formation from ternary mixtures during the evaporation of one component, European Physical Journal Special Topics 228 (2019) 55-68.

[5] P. C. Fife, Pattern formation in gradient systems, in: B. Fielder (Ed.), Handbook of Dynamical Systems, Elsevier Science, Amsterdam, The Netherlands, vol. 2, pp. 677-722.

[6] N. Alikakos, P. W. Bates, G. Fusco, Slow motion for the cahn-hilliard equation in one space dimension, Journal of Differential Equations 90 (1991) $81-135$.

[7] S. M. Allen, J. W. Cahn, A microscopic theory for antiphase boundary motion and its application to antiphase domain coarsening, Acta Metallurgica 27 (1979) 1085-1095. 
[8] E. N. M. Cirillo, N. Ianiro, G. Sciarra, Phase coexistence in consolidating porous media, Physical Review E 81 (2010) 061121.

[9] E. N. M. Cirillo, N. Ianiro, G. Sciarra, Allen-cahn and cahn-hilliard-like equations for dissipative dynamics of saturated porous media, Journal of the Mechanics and Physics of Solids 61 (2013) 1080-1114.

[10] G. Sciarra, Phase field modeling of partially saturated deformable porous media, Journal of the Mechanics and Physics of Solids 94 (2016) 230-256.

[11] R. Benzi, M. Sbragaglia, M. Bernaschi, S. Succi, Phase-field model of longtime glasslike relaxation in binary fluid mixtures, Physical Review Letters 106 (2011) 164501.

[12] L. Cueto-Felgueroso, R. Juanes, Macroscopic phase-field model of partial wetting: Bubbles in a capillary tube, Physical Review Letters 108 (2012) 144502.

[13] E. N. M. Cirillo, N. Ianiro, G. Sciarra, Compacton formation under allencahn dynamics, Proc. R. Soc. A 472 (2016) 20150852.

[14] E. N. M. Cirillo, G. Saccomandi, G. Sciarra, Compact structure as true non-linear phenomena, Mathematics in Engineering 1 (2019) 434-446.

[15] M. Destrade, G. Gaeta, G. Saccomandi, Weierstrass' criterion and compact solitary waves, Physical Review E 75 (2007) 047601.

[16] P. Rosenau, A. Zilburg, Compactons, Journal of Physics A 51 (2018) 343001.

[17] N. Alikakos, G. Fusco, On the connection problem for potentials with several global minima, Indiana University Mathematics Journal 57 (2008) $1871-1906$.

[18] J. Carr, M. E. Gurtin, M. Slemrod, Structured phase transitions on a finite interval, Archive for Rational Mechanics and Analysis 86 (1984) 317-351. 
[19] P. C. Fife, J. B. McLeod, The approach of solutions of nonlinear diffusion equations to travelling front solutions, Arch. Ration. Mech. Anal. 65 (1977) $335-361$.

[20] L. Bertini, S. Brassesco, P. Buttà, Soft and hard wall in a stochastic reaction diffusion equation, Arch. Rational Mech. Anal. 190 (2008) 307-345.

[21] L. Rey-Bellet, Ergodic properties of markov processes, in: Open quantum systems ii, 1-39, ecture notes in math. 1881, springer, berlin, 2006.

[22] A. Friedman, Partial differential equations of parabolic type, prentice-hall, inc., englewood cliffs, n.j. 1964.

[23] M. Meyries, J. D. M. Rademacher, E. Siero, Quasi-linear parabolic reaction-diffusion systems: A user's guide to well-posedness, spectra, and stability of travelling waves, SIAM J. Appl. Dyn. Syst. 13 (2014) 249-275.

[24] O. A. Ladyženskaja, V. A. Solonnikov, N. N. Ural'ceva, Linear and quasilinear equations of parabolic type, translations of mathematical monographs vol. 23, american mathematical society, providence, 1968. 